\title{
A TOPOLOGICAL APPROACH FOR DETERMINING THE UNIQUENESS OF THE DC SOLUTIONS IN MOS-TRANSISTOR CIRCUITS
}

\author{
Arturo Sarmiento-Reyes ${ }^{1}$, Luis Hernández-Martínez ${ }^{2}$ and Hector Vázquez-Leal ${ }^{2}$ \\ ${ }^{1}$ Delft University of Technology, ITS, Electronics Research Lab, Mekelweg 4, 2628CD Delft, The \\ Netherlands. \\ ${ }^{2}$ INAOE, Electronics Department, CAD Group P.O. Box 51, 72000 Puebla, Pue., Mexico. \\ Email: luish@inaoep.mx
}

\begin{abstract}
This paper presents a method focused on assessing the uniqueness of the DC solution of mos-transistor networks. The method is based on purely topological conditions of the network graph, and it is based on identifying positive feedback structures (PFSs) by resorting to the definitions given by Hasler. The topological conditions for the existence of PFSs at nullator \& norator level are established.
\end{abstract}

\section{INTRODUCTION}

$\mathrm{T}$ HE complete solution to the DC general problem of nonlinear circuits must include three aspects [1]: determining whether or not the uniqueness of the DC solution is guaranteed, establishing an upper bound on the number of DC solutions, and as last step calculating all of them, as well as determining their stability conditions. Several works have been focussed on the first problem [2], which is strongly bound with two aspects of the circuit: the circuit topology and the values of the circuit parameters.

A line of research developed in recent years was focussed on determining the relationships between the topology of a circuit and its functionality, which has derived in a deeper knowledge in the general problem of nonlinear resistive circuits [2], [3]. In the works of Hasler and Fosséprez [4], [5], [6], [7], a topological criterion for the existence and uniqueness of the solution of nonlinear circuits has been proposed. This criterion is based on two concepts of Graph Theory: the pair of conjugate trees and the uniform partial orientation of the resistors.

However, the task of finding the tree of a given graph has complexity nature, which

A. Sarmiento Reyes is on sabbatical leave from the National Institute of Astrophysics Optics and Electronics (INAOE).

0-7803-7057-0/01/\$10.00 C2001 IEEE. makes this criterion not useful for practical circuits.

In this context, the main contribution of our work is to determine the existence of the PFSs in a systematic way by resorting to a slightly modified form of the definition of the conjugated tree pair introduced by Hasler.

\section{EXISTENCE AND UNIQUENESS CRITERIA.}

The scope of our analysis is restricted to certain types of basic circuits elements: resistors $(R)$, voltage sources $(V)$, current sources $(I)$, nullators $(O)$ and norators $(P)$. The norators and nullators are supposed to be present in the circuit in equal numbers [8]. Other circuit elements can be modelled by connections of these basic elements, e.g. operational amplifiers and transistors.

Herein, we retake two definitions from [9] in order to set up the further development of our method:

\section{DEFINITION 1}

A nonlinear resistive circuit structure is a graph whose branches are labeled with the following six element types

- independent voltage sources

- independent current sources

- $V$-resistors (voltage controlled)

- I-resistors (current controlled)

- nullators

- norators

A circuit $C$ has a structure $S$ if the graph of $C$ and $S$ coincide and if $C$ has elements of the type prescribed by $S$ on each branch.

\section{DEFINITION 2}

Two trees $t^{\prime}$ and $t^{\prime \prime}$ of a nonlinear resistive circuit structure constitute a pair of conjugate trees if 
- $t^{\prime}$ is composed of all norators, all voltage sources and a subset of resistors, and

- $t^{\prime \prime}$ is composed of all nullators, all voltage sources and the same resistors as in $t^{\prime}$.

The subsets of the resistors may also be empty or contain all resistors.

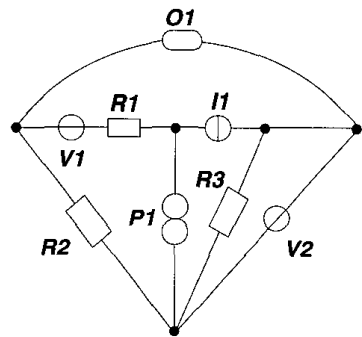

Fig. 1. A linear circuit.

In order to illustrate these concepts, consider the linear circuit depicted in the Figure 1, which fulfills the Definition 1.

The conjugate tree pair is formed according to the Definition 2. On one hand, the tree $t^{\prime}$ is formed by the norator, both voltage sources and the resistor $\mathrm{R} 1$, as given in the Figure 2(a). On the other side, the tree $t^{\prime \prime}$ is formed by the nullator, both voltage sources and the same resistor, as given in the Figure 2(b).

\section{DEFINITION 3}

A partial orientation of the resistors is uniform, if

- every oriented resistor is part of a uniform loop composed only of oriented resistors, norators and voltage sources, and

- every oriented resistors is part of a uniform cutset composed only of oriented resistors, norators.

This definition is illustrated in the Figure 3, where the simplest array of cutset and loop exhibits a compatible $i-v$ orientation.

The last two definitions determine whether a given circuit may or not posses multiple DC solutions.

\section{Positive feedback structures}

In [10], [11], a fundamental result concerning the uniqueness of the DC solution of transistor circuits has been established by identifying the presence of a PFSs embedded in the circuit

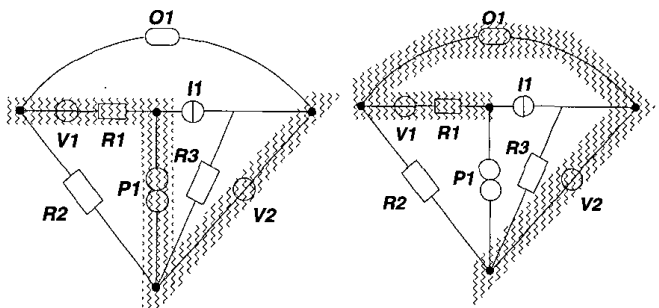

Fig. 2. Pair of conjugate trees.

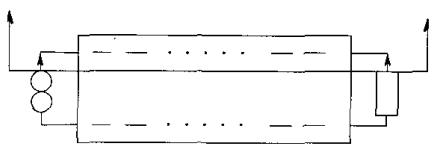

(a)

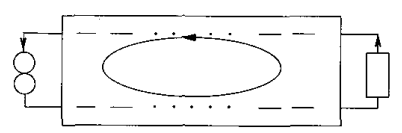

(b)

Fig. 3. The simplest pair of compatible $i-v$ orientations.

topology, which has the flip-flop-like structure shown in the Figure 4.

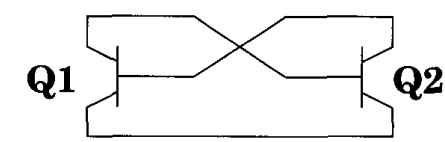

Fig. 4. BJT Positive Feedback Structure.

\subsection{Mos-Transistor model}

The circuit equivalent used to model the mostransistor is shown in the Figure 5. It corresponds to a $C$ structure introduced by Hasler. In the following, we shall see how two mostransistors, that are modelled by the Hasler structure, may form a positive feedback structure.

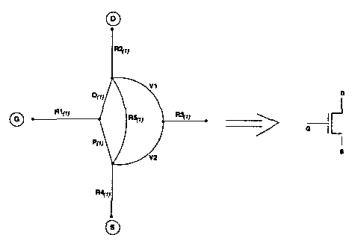

Fig. 5. Mos-transistor model: C structure 


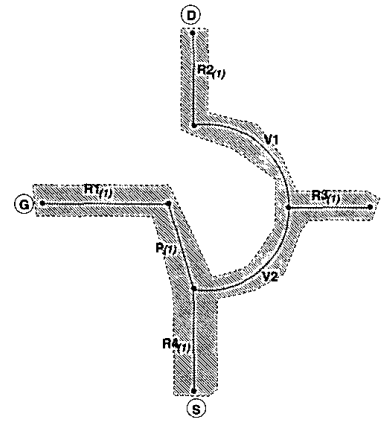

(a)

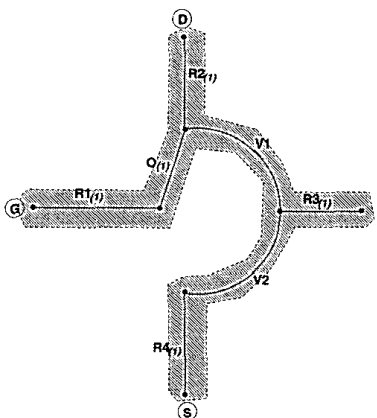

(c)
Fig. 6. (a) Tree $\left(\boldsymbol{t}^{\prime}\right)$, and (b) Conjugated tree $\left(\boldsymbol{t}^{\prime \prime}\right)$, of the BJT C structure

\section{Positive feedback Structures \& TREE-PAIRS}

In order to determine the conditions for the uniqueness of the DC solution by resorting to the Definitions 1 and 2, the next concepts are introduced.

Mos-Transistor tree. It is the tree of the transistor model from the Figure 5, which must be formed as follows:

$$
\boldsymbol{t}^{\prime}=\{\mathrm{P}, \mathrm{R} 1, \mathrm{R} 2, \mathrm{R} 3, \mathrm{R} 4, \mathrm{~V} 1, \mathrm{~V} 2\}
$$

as given in the Figure 6(a).

Mos-Transistor conjugated tree. It is the tree of the transistor model from the Figure 5(b), which must be formed as follows:

$$
\boldsymbol{t}^{\prime}=\{\mathrm{O}, \mathrm{R} 1, \mathrm{R} 2, \mathrm{R} 3, \mathrm{R} 4, \mathrm{~V} 1, \mathrm{~V} 2\}
$$

as given in the Figure 6(b).

\subsection{A simple result on the mos-transistor model}

A first - and rather trivial - result consists in the fact that the conjugated trees of the mostransistor model do not have a pair of uniform $i$ - $u$ partial orientation, which means that a single mos-transistor does not posses multiple DC solutions.

This is necessary to be highlighted because the graphs of $\boldsymbol{t}^{\prime}$ and $\boldsymbol{t}^{\prime \prime}$ will be used to generate a PFS.

\subsection{Forming a PFS}

For a given pair of mos-transistors $\left(M_{1}, M_{2}\right)$, two pairs of subgraphs arise, namely $\left(t_{1}^{\prime}, t_{1}^{\prime \prime}\right)$ and $\left(t_{2}^{\prime}, t_{2}^{\prime \prime}\right)$. Another subgraph is the graph associated to the positive linear resistors of the circuit, and it is denoted as $G_{R}$. The Figure 7 schematically shows these subgraphs.

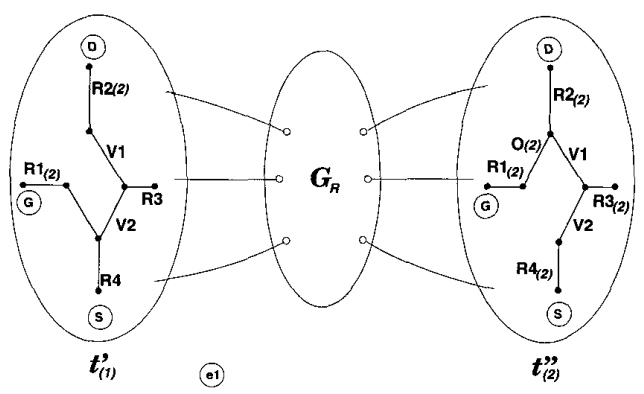

Fig. 7. Forming a PFS with the conjugated tree pair.

The main result can be stated as follows:

A positive feedback structure is obtained through applying the next graph operations on the tree-pair and the resistor subgraphs:

$$
H_{1} \triangleq t_{1}^{\prime} \cup t_{2}^{\prime \prime} \cup \hat{G}_{R}
$$

and

$$
H_{2} \triangleq t_{1}^{\prime \prime} \cup t_{2}^{\prime} \cup \hat{G}_{R}
$$

where $\hat{G}_{R}$ is the modified resistor graph obtained by deleting and/or contracting some resistor branches, such that the terminal pairs $\left(S_{1}, S_{2}\right),\left(D_{1}, G_{2}\right)$ and $\left(G_{1}, D_{2}\right)$ get connected, as depicted in the Figure 8 . The presence of such a graph embedded in the network topology is an indication that the circuit possesses multiple DC solutions.

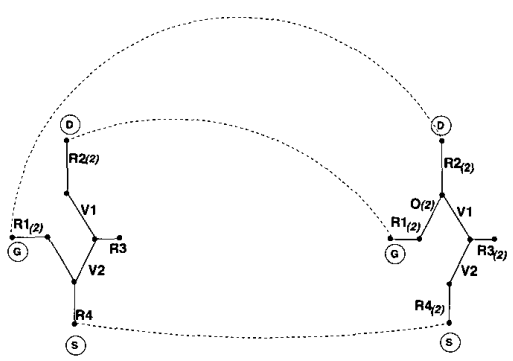

Fig. 8. A PFS from the conjugated tree pair.

\subsection{Procedure for detecting a PFS}

The method for identifying PFSs generated by the interconnection of the conjugated tree 
pairs of the mos-transistor models can be recast as:

1. Short-circuit all voltage sources and opencircuit all current sources.

2. All resistors are replaced by a non-directed edge.

3. Replace the mos-transistor by the graph equivalents of $\boldsymbol{t}^{\prime}$ and $\boldsymbol{t}^{\prime \prime}$.

4. Apply short-circuit and/or open-circuit operations to some resistors in $\boldsymbol{G}_{R}$.

5. If there exists a positive feedback structure then the circuit possesses multiple DC solutions.

6. Else there exists a positive feedback structure and also there not exists a pair of compatible $i-v$ orientations then the uniqueness is guarented.

\section{EXAMPLE}

In this section, the method is illustrated through the circuit shown in the Figure 9(a). The graph associated to the network without voltage sources is given in the Figure 9(b).

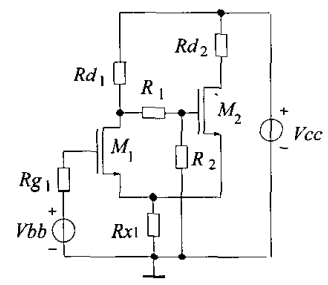

(a)

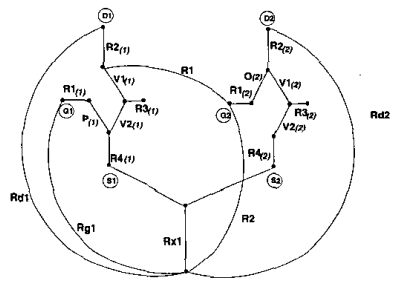

(b)
Fig. 9. The example circuit and the graph of the dead network.

Therefore, the structure producing multiple DC operating points (FPS) can be obtained by applying:

$\mathcal{S}\left(R 1, R 2, R_{g 1}, R 1_{1}, R 4_{1}, R 1_{2}, R 4_{2}, R 3_{2}, V 1_{2}, V 2_{2}\right)$

and

$$
\mathcal{O}\left(R_{x e 1}, R_{d 1}, R_{d 2}, R 2_{1}, R 3_{1}, R 2_{2}, V 1_{1}, V 2_{1}\right)
$$

where $\mathcal{S}(\bullet)$ and $\mathcal{O}(\bullet)$ stands for short-circuit and open-circuit operations respectively. The result is shown in the Figure 10.

\section{Conclusions}

A method based on purely topological considerations has been developed in order to deter-

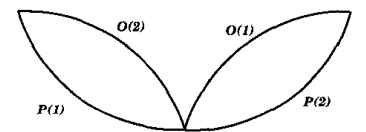

Fig. 10. Positive Feedback Structure

mine the uniqueness of the DC operating point. It resorts to the conjugated tree pair concept in order to detect PFSs, which are the origin of multiple DC solutions.

\section{REFERENCES}

[1] Sarmiento Reyes L. A., A partition method for the determination of multiple $D C$ operating points, $\mathrm{Ph}$.D. thesis, Delft University of Technology, May 1994.

[2] Tetsuo Nishi, "On the number of solutions of a class of nonlinear resistive circuit," Proceedings of the IEEE International Symposium on Circuits and Systems, Singapore, pp. 766-769, 1991.

[3] Tetsuo Nishi and Leon O. Chua, "Topological criteria for nonlinear resistive circuits containing controlled sources to have a unique solution," IEEE Transactions on Circuits and Systems, vol. CAS31, no. 8, pp. 722-741, August 1984.

[4] Martin Hasler, "Monotonic dependence in mostransistor circuits," 10th European Conference on Circuit Theory and Design, Denmark, pp. 11231131, 1991.

[5] Marc Fosséprez, Martin Hasler, and Claudia Schnetzler, "On the number of solutions of piecewise-linear resistive circuits," IEEE Transactions on Circuits and Systems, vol. 18, no. 3, pp. 393-402, March 1989.

[6] Martin Hasler, "Non-linear non-reciprocal resistive circuits with structurally unique solution," International Journal on Circuit Theory and Applications, vol. 14, pp. 237-262, 1986.

[7] Marc Fosséprez and Martin Hasler, "Resistive circuit topologies that admit several solutions," International Journal on Circuit Theory and Applications, vol. 18, no. 6, pp. 625-638, December 1990.

[8] B.D.H. Tellegen, "On nullators and norators," IEEE Trans. on Circuits Theory, , no. 4, pp. 466$469,1966$.

[9] Marc Fosséprez, Non-linear Circuits, John Wiley \& sons, 1989.

[10] R. O. Nielsen and A. N. Willson, "A fundamental result concerning the topology of transistor circuits with multiple equilibria," Proceedings of the IEEE, vol. 68, no. 2, pp. 196-208, February 1980.

[11] I. W. Sandberg and A. N. Willson Jr., "Topological criteria for establishing the uniqueness of solutions to the DC equations of transistor networks." IEEE Transactions on Circuits and Systems, vol. CAS-24, no. 7, pp. 349-362, July 1977. 